Archive for

Organic Chemistry

Arkivoc 2018, part iii, $184-190$

\title{
Oxidative Route to Pyrroloisoquinoline-2,3-dione
}

\author{
Hari K. Kadam *a and Santosh G. Tilve ${ }^{\text {b }}$ \\ ${ }^{a}$ Department of Chemistry, St. Xavier's College, Mapusa, Goa-403507 India . \\ ${ }^{b}$ Department of Chemistry, Goa University, Taleigao plateau, Goa-403206 India \\ Email: harikadam05@gmail.com
}

Received 12-12-2017

Accepted 01-28-2018

Published on line 02-25-2018

\section{Abstract}

We herein report an efficient constructive method for synthesis of structurally important Pyrroloisoquinoline2,3-dione from dihydroisoquinoline through oxidative cyclisation. Process is optimised to give best efficiency at gram scale and laborious purification techniques such as column chromatography or recrystallisation were avoided in all steps further featuring uniqueness of this method as compared to the available literature.
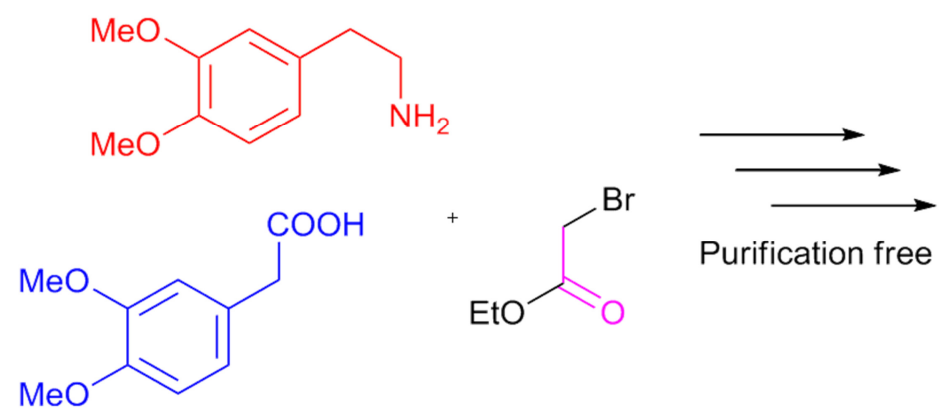

Purification free method<smiles>CCOC(=O)CBr</smiles><smiles>COc1ccc(C2=C3c4cc(OC)c(OC)cc4CCN3C(=O)C2=O)cc1OC</smiles>

Dihydropyrrolo[2,1-a]isoquinoline-2,3-dione

Keywords: Isoquinoline, pyrrole, aerobic oxidation, synthesis, green chemistry, dione 


\section{Introduction}

Multiannular heterocycles are frequently encountered in several anticancer, antiviral, antibacterial compounds and naturally occurring microbial or marine metabolites or phytochemicals. ${ }^{1-4}$<smiles>CCCN1C(=O)C(=O)c2c3c(OC)cc1c2-c1ccccc1-3</smiles><smiles></smiles>

Lettowianthine, Annonbraine<smiles>COc1c(OC)c2c3c4c(c5ccccc5c-2c1OC)C(=O)C(=O)N4CC3</smiles>

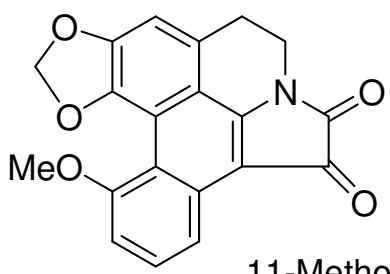<smiles></smiles><smiles>COc1ccc(C2=C3c4cc(OC)c(OC)cc4CCN3C(=O)C2=O)cc1OC</smiles>

Dihydropyrrolo[2,1-a]isoquinoline-2,3-dione

Figure 1. Naturally occurring pyrroloquinoline-1,2-diones and pyrroloisoquinoline-2,3-dione under study.

Pyrroloquinoline-1,2-diones ${ }^{5-13}$ are observed in natural compounds such as telisatin A and B, laurodionine, annonbraine, methoxylettowianthine as depicted in Figure 1.

During our studies towards medicinally important organic heterocycles, ${ }^{14-16}$ we encountered a route for synthesis of Pyrroloisoquinoline-2,3-dione ${ }^{17-19}$ which are structurally similar to pyrroloquinoline-1,2-diones. In this paper we describe an efficient, gram scale, purification free method for synthesis of pyrroloisoquinoline2,3-dione 1 from dihydroisoquinoline 2 through oxidative cyclisation.

\section{Results and Discussion}

We began by preparing secondary amide $\mathbf{3}$ of homoveratric acid and homoveratryl amine by two alternate methods ${ }^{20}$ via acid chloride and via DCC coupling as described in scheme 1. 


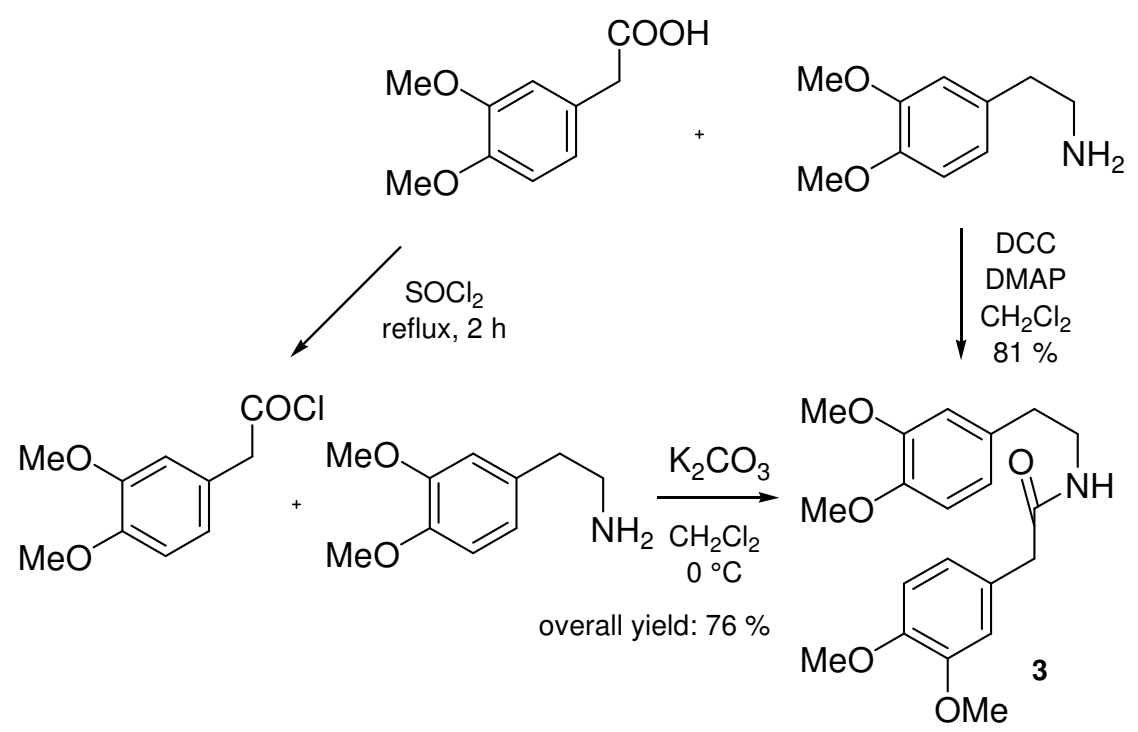

Scheme 1. Synthesis of secondary amide 3.

Dihydroisoquinoline 2 was prepared by Bischler-Napieralski reaction ${ }^{20-25}$ (Scheme 2) on amide 3 . Ethyl bromoacetate with dihydroisoquinoline $\mathbf{2}$ gave the corresponding salt which was in situ treated with triethylamine in aerobic refluxing condition. The overall product obtained was identified to be pyrroloisoquinoline-2,3-dione 1 . With process optimisation to give best efficiency, (Scheme 3) laborious purification techniques such as column chromatography or recrystallisation were avoided in all steps further featuring uniqueness of this method as compared to the available literature.<smiles>COc1ccc(CC2=NCCc3cc(OC)c(OC)cc32)cc1OC</smiles>

Scheme 2. Preparation of dihydroisoquinoline 2.<smiles>COc1ccc(CC2=NCCc3cc(OC)c(OC)cc32)cc1OC</smiles>

1) Ethyl bromoacetate, Toluene, r.t. $6 \mathrm{~h}$<smiles>COc1ccc(C2=C3c4cc(OC)c(OC)cc4CCN3C(=O)C2=O)cc1OC</smiles>

Scheme 3. Preparation of pyrroloisoquinoline-2,3-dione 1.

On successful method development, we also propose herein a probable mechanistic pathway as described in scheme 4 for this constructive transformation. Dihydroisoquinoline $\mathbf{2}$ reacts with ethyl bromoacetate to 
form the quaternary ammonium salt. Addition of base gives enamine ester which undergoes intramolecular cyclisation to give pyrroloisoquinoline.

Further presence of base gives azomethine which undergoes aerobic oxidation to directly give pyrroloisoquinoline-2,3-dione 1.

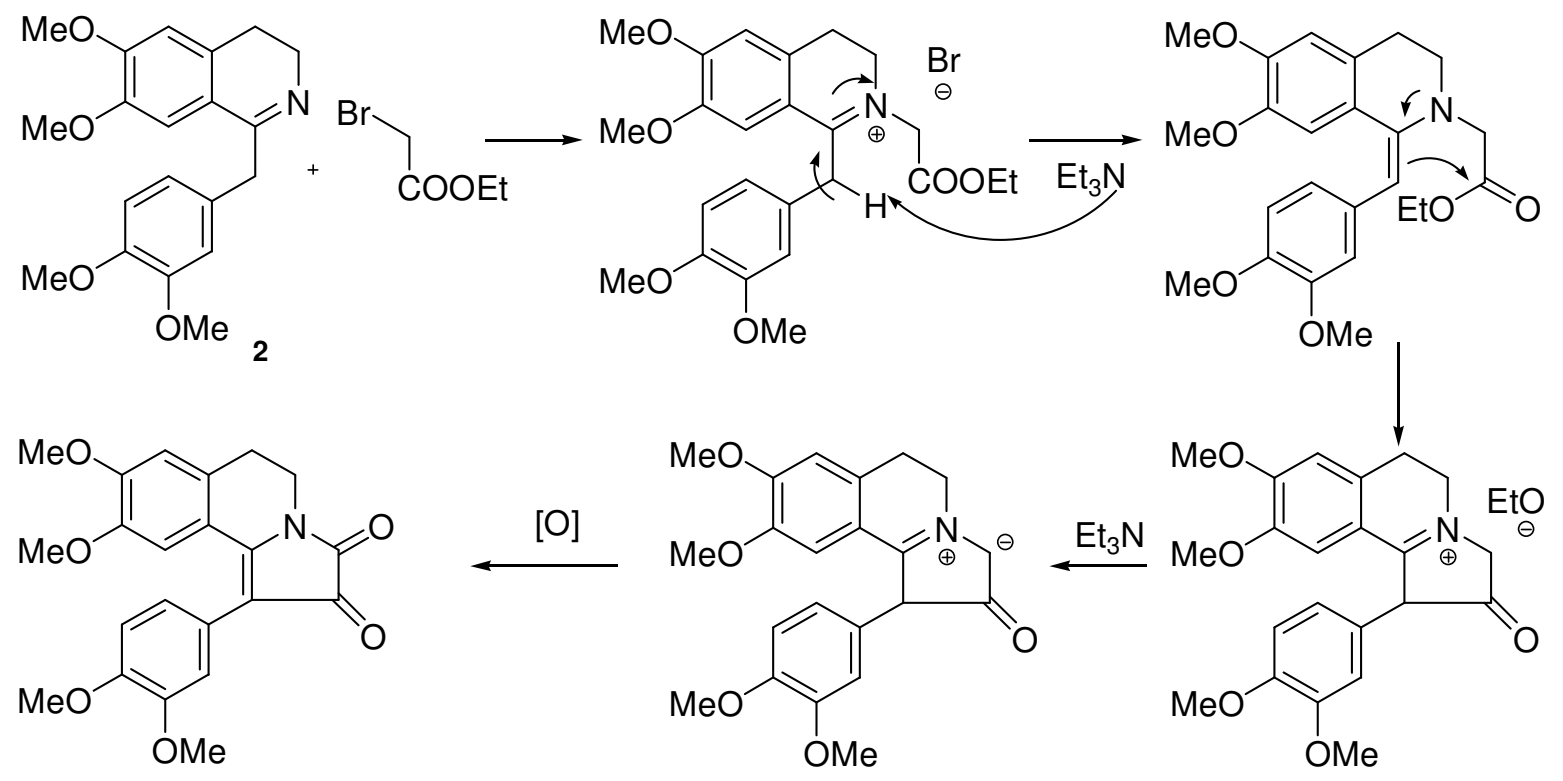

1

Scheme 4. Probable mechanism for transformation of dihydroisoquinoline to pyrroloisoquinoline-2,3-dione.

\section{Conclusions}

In conclusion, we have developed an efficient constructive method for synthesis of structurally important Pyrroloisoquinoline-2,3-dione from dihydroisoquinoline through oxidative cyclisation at gram scale. Laborious purification techniques such as column chromatography or recrystallisation were avoided in all steps further featuring uniqueness of this method.

\section{Experimental Section}

General. Reagents were purchased from Sigma-Aldrich and were used without further purification. IR spectra were recorded with Shimadzu FTIR instrument. ${ }^{1} \mathrm{H} \&{ }^{13} \mathrm{C} N M R$ spectra were recorded in DMSO-d 6 with Bruker AVANCE $400 \mathrm{MHz}$ NMR Spectrometer. LCMS were recorded with Shimadzu LCMS instrument. HRMS were recorded with a MicroMass ESQTOF.

$\mathbf{N}$-(3,4-Dimethoxyphenethyl)-2-(3,4-dimethoxyphenyl)acetamide (3). (a) $\mathrm{SOCl}_{2}$ method: Homoveratric acid (5 $\mathrm{g}, 25.5 \mathrm{mmol})$ was added to freshly distilled thionyl chloride $(15 \mathrm{~mL})$ and refluxed at $100{ }^{\circ} \mathrm{C}$ for $3 \mathrm{~h}$. Excess thionyl chloride was removed from reaction mixture by distillation and dry $\mathrm{CHCl}_{3}(10 \mathrm{~mL})$ was added. This solution of acid chloride was added dropwise with stirring to an ice cold solution of homoveratryl amine (4.16 $\mathrm{g}, 23.0 \mathrm{mmol})$ and $\mathrm{K}_{2} \mathrm{CO}_{3}(5.53 \mathrm{~g} 40 \mathrm{mmol})$ in dry $\mathrm{CHCl}_{3}(20 \mathrm{~mL})$. This mixture was stirred for $12 \mathrm{~h}$ from $0{ }^{\circ} \mathrm{C}$ to 
r.t. Solvent was removed under vacuum and distilled water $(50 \mathrm{~mL})$ was added. The solid thus obtained was filtered and washed with water $(20 \mathrm{~mL} \times 3)$ and dried under vacuum. Analytically pure product 3 was obtained as white amorphous solid in $76 \%(6.28 \mathrm{~g})$ yield without any further purification. White amorphous solid, mp: 124-125 ${ }^{\circ} \mathrm{C}$. [lit. $\mathrm{mp} 124-125^{\circ} \mathrm{C}{ }^{20} \mathrm{IR}(\mathrm{KBr}): v_{\max } 3325,2960,1641,1589,1517,1028 \mathrm{~cm}^{-1} .{ }^{1} \mathrm{H} \mathrm{NMR}(400 \mathrm{MHz}$, $\left.\mathrm{CDCl}_{3}\right): \delta 2.60(\mathrm{t}, J 6.8 \mathrm{~Hz}, 2 \mathrm{H}), 3.36(\mathrm{~m}, 2 \mathrm{H}), 3.41(\mathrm{~s}, 2 \mathrm{H}), 3.75(\mathrm{~s}, 3 \mathrm{H}), 3.76(\mathrm{~s}, 3 \mathrm{H}), 3.79(\mathrm{~s}, 3 \mathrm{H}), 3.81(\mathrm{~s}, 3 \mathrm{H}), 5.37$ (br s, 1H), 6.45 (dd, J 8.4, 1.6 Hz, 1H), $6.54(\mathrm{~d}, \mathrm{~J} 1.6 \mathrm{~Hz}, 1 \mathrm{H}), 6.62(\mathrm{~m}, 3 \mathrm{H}), 6.73(\mathrm{~m}, 1 \mathrm{H}) \mathrm{ppm} .{ }^{13} \mathrm{C}$ NMR \& DEPT $(100 \mathrm{MHz}, \mathrm{CDCl})$ ): $\delta 34.97\left(\mathrm{CH}_{2}\right), 40.71\left(\mathrm{CH}_{2}\right), 43.42\left(\mathrm{CH}_{2}\right), 55.81\left(\mathrm{CH}_{3}\right), 55.84\left(2 \mathrm{x} \mathrm{CH}_{3}\right), 55.89\left(\mathrm{CH}_{3}\right), 111.08(\mathrm{CH})$, $111.39(\mathrm{CH}), 111.64(\mathrm{CH}), 112.36(\mathrm{CH}), 120.56(\mathrm{CH}), 121.59(\mathrm{CH}), 127.14(\mathrm{Cq}), 131.00(\mathrm{Cq}), 147.61(\mathrm{Cq}), 148.27$ (Cq), $148.91(\mathrm{Cq}), 149.22(\mathrm{Cq}), 171.30(\mathrm{Cq}) \mathrm{ppm}$.

(b) DCC coupling method: Homoveratric acid (5 g, $25.5 \mathrm{mmol})$, homoveratryl amine (4.62 $\mathrm{g}, 25.5 \mathrm{mmol})$ and DMAP $(0.05 \mathrm{~g})$ were added in dry $\mathrm{CH}_{2} \mathrm{Cl}_{2}(25 \mathrm{~mL})$ and cooled to $0{ }^{\circ} \mathrm{C}$. To this mixture, $\mathrm{DCC}(6.19 \mathrm{~g}, 30 \mathrm{mmol})$ was added and stirred from $0{ }^{\circ} \mathrm{C}$ to r.t. for $24 \mathrm{~h}$. Water $(1 \mathrm{~mL})$ and dioxane $(2 \mathrm{~mL})$ was added to this and stirred for $2 \mathrm{~h}$. Solvent was removed under vacuum, $\mathrm{CH}_{2} \mathrm{Cl}_{2}(25 \mathrm{~mL})$ was added, cooled to $0{ }^{\circ} \mathrm{C}$ and filtered. The filtrate was again cooled to $0{ }^{\circ} \mathrm{C}$ and filtered. The solvent was removed under vacuum and product 3 was obtained as white solid in $81 \%$ ( $7.41 \mathrm{~g}$ ) yield without any further purification.

1-(3,4-Dimethoxybenzyl)-6,7-dimethoxy-3,4-dihydroisoquinoline (2). Amide 3 (7.18 g, $20 \mathrm{mmol}$ ) was dissolved in dry toluene $(10 \mathrm{~mL})$ and freshly distilled $\mathrm{POCl}_{3}(5 \mathrm{~mL})$ was added slowly and refluxed for $4 \mathrm{~h}$. The reaction mixture was then poured in ice and basified by cooled aq. $\mathrm{NaOH}$ solution (10 N) until pH 14 . Dihydroisoquinoline 2 was then extracted in $\mathrm{CH}_{2} \mathrm{Cl}_{2}(20 \mathrm{~mL} \times 2)$, dried by passing through anhy. $\mathrm{Na}_{2} \mathrm{SO}_{4}$ and concentrated under vacuum to give $71 \%(4.84 \mathrm{~g})$ yield without any further purification. Viscous oil. ${ }^{20}{ }^{1} \mathrm{H} N M R$ $\left(400 \mathrm{MHz}, \mathrm{CDCl}_{3}\right)$ : $\delta 1.89(\mathrm{~m}, 2 \mathrm{H}), 2.76(\mathrm{t}, \mathrm{J} 8.0 \mathrm{~Hz}, 2 \mathrm{H}), 3.74(\mathrm{~s}, 3 \mathrm{H}), 3.76(\mathrm{~s}, 3 \mathrm{H}), 3.79(\mathrm{~s}, 3 \mathrm{H}), 3.86(\mathrm{~s}, 3 \mathrm{H}), 4.20$ $(\mathrm{s}, 2 \mathrm{H}), 6.64(\mathrm{~s}, 1 \mathrm{H}), 6.69(\mathrm{~d}, \mathrm{~J} 8.4 \mathrm{~Hz}, 1 \mathrm{H}), 6.78(\mathrm{~m}, 1 \mathrm{H}), 6.95(\mathrm{~s}, 1 \mathrm{H}), 7.09(\mathrm{~s}, 1 \mathrm{H}) \mathrm{ppm} .{ }^{13} \mathrm{C}$ NMR \& DEPT $(100$ $\left.\mathrm{MHz}, \mathrm{CDCl}_{3}\right): \delta 24.95\left(\mathrm{CH}_{2}\right), 33.20\left(\mathrm{CH}_{2}\right), 49.11\left(\mathrm{CH}_{2}\right), 55.89\left(\mathrm{CH}_{3}\right), 56.04\left(\mathrm{CH}_{3}\right), 56.05\left(\mathrm{CH}_{3}\right), 56.12\left(\mathrm{CH}_{3}\right), 110.02$ $(\mathrm{CH}), 112.20(\mathrm{CH}), 112.04(\mathrm{CH}), 121.16(\mathrm{CH}), 126.64(\mathrm{CH}), 131.09(\mathrm{Cq}), 133.89(\mathrm{Cq}), 147.66(\mathrm{Cq}), 149.68(\mathrm{Cq})$, $151.71(\mathrm{Cq}), 154.19(\mathrm{Cq}), 156.13(\mathrm{Cq}), 164.69$ (Cq) ppm.

\section{1-(3,4-Dimethoxyphenyl)-8,9-dimethoxy-5,6-dihydropyrrolo[2,1-a]isoquinoline-2,3-dione (1).}

Dihydroisoquinoline $2(1.1 \mathrm{~g}, 3.22 \mathrm{mmol})$ in dry toluene $(25 \mathrm{~mL})$ was cooled to $0{ }^{\circ} \mathrm{C}$ and Ethyl bromoacetate $(0.6 \mathrm{~g}, 3.4 \mathrm{mmol})$ in dry toluene $(5 \mathrm{~mL})$ was added and stirred from $0{ }^{\circ} \mathrm{C}$ to r.t. for $6 \mathrm{~h}$. Further mixture was cooled to $0{ }^{\circ} \mathrm{C}$ and insoluble salt was isolated by decanting. To this salt, triethylamine $(10 \mathrm{~mL})$ was added and refluxed in air for $12 \mathrm{~h}$. Finally excess triethylamine was removed under vacuum and ice cold distilled water $(50 \mathrm{~mL})$ was added. The solid product thus obtained was filtered and washed with water $(20 \mathrm{~mL} \mathrm{X} \mathrm{3)} \mathrm{and} \mathrm{dried}$ under vacuum. Analytically pure pyrroloisoquinoline 1 was obtained as wine red solid in $85 \%(1.08 \mathrm{~g})$ yield without any further purification. Wine red solid, $\mathrm{mp}: 176-178{ }^{\circ} \mathrm{C}, \mathrm{IR}(\mathrm{KBr}): \mathrm{v}_{\max } 3021,1728,1705,1624,1445$ $\mathrm{cm}^{-1} .{ }^{1} \mathrm{H}$ NMR $\left(400 \mathrm{MHz}\right.$, DMSO- $\left.d_{6}\right): \delta 3.06(\mathrm{t}, J 6.0 \mathrm{~Hz}, 2 \mathrm{H}), 3.25(\mathrm{~s}, 3 \mathrm{H}), 3.68(\mathrm{~s}, 3 \mathrm{H}), 3.70(\mathrm{t}, J 6.4 \mathrm{~Hz}, 2 \mathrm{H}), 3.77$ $(\mathrm{s}, 3 \mathrm{H}), 3.86(\mathrm{~s}, 3 \mathrm{H}), 6.83(\mathrm{~m}, 1 \mathrm{H}), 6.85(\mathrm{~s}, 1 \mathrm{H}), 6.92(\mathrm{~s}, 1 \mathrm{H}), 7.03(\mathrm{~d}, J=8.4 \mathrm{~Hz}, 1 \mathrm{H}), 7.09(\mathrm{~s}, 1 \mathrm{H}) \mathrm{ppm} .{ }^{13} \mathrm{C} \mathrm{NMR}$ (100 MHz, DMSO-d $\left.)_{6}\right): \delta 27.55\left(\mathrm{CH}_{2}\right), 35.90\left(\mathrm{CH}_{2}\right), 54.62\left(\mathrm{CH}_{3}\right), 55.53\left(\mathrm{CH}_{3}\right), 55.61\left(\mathrm{CH}_{3}\right), 55.94\left(\mathrm{CH}_{3}\right), 107.24$ $(\mathrm{Cq}), 111.45(\mathrm{CH}), 112.02(\mathrm{CH}), 112.16(\mathrm{CH}), 113.49(\mathrm{CH}), 115.97(\mathrm{Cq}), 122.56(\mathrm{CH}), 122.94(\mathrm{Cq}), 133.84(\mathrm{Cq})$, $146.98(\mathrm{Cq}), 148.37(\mathrm{Cq}), 148.82(\mathrm{Cq}), 153.14(\mathrm{Cq}), 157.05(\mathrm{Cq}), 158.00(\mathrm{Cq}), 182.85(\mathrm{Cq}) \mathrm{ppm}$. LCMS (m/z): $[\mathrm{M}+\mathrm{H}]^{+}$395.9. HRMS (m/z): calculated for $\mathrm{C}_{22} \mathrm{H}_{21} \mathrm{NO}_{6} \mathrm{Na}[\mathrm{M}+\mathrm{Na}]^{+}:$418.1267; found : 418.1289 . 


\section{Acknowledgements}

Authors thank Science \& Engineering Research Board (SERB), Department of Science \& Technology, New Delhi for funding. Authors acknowledge Indian Institute of Science (IISC), Bangalore for HRMS analysis.

\section{Supplementary Material}

Supplementary data $\left({ }^{1} \mathrm{H}\right.$ NMR, ${ }^{13} \mathrm{C}$ NMR and DEPT spectra of all the products) associated with this article can be found, in the online version.

\section{References}

1. Liu, B.; Jian, L.; Chen, G.; Song, X.; Han, C.; Wang, J. Chemistry of Natural Compounds 2014, 49, 1172. https://doi.org/10.1007/s10600-014-0855-6

2. Polygalova, N. N.; Mikhailovskii, A. G.; Vikhareva, E. V.; Vakhrin, M. I. Chemistry of Heterocyclic Compounds 2007, 43, 900. https://doi.org/10.1007/s10593-007-0142-6

3. Hwang, T. L.; Wu, Y. C.; Yeh, S. H.; Kuo, R. Y., Biochemical Pharmacology 2004, 69, 65. https://doi.org/10.1016/i.bcp.2004.09.010

4. Wu, C.-C.; Wang, W.-Y.; Kuo, R.-Y.; Chang, F.-R.; Wu, Y.-C. Eur. J. Pharmacology 2004, 483, 187. https://doi.org/10.1016/i.ejphar.2003.10.046

5. Chang, F.-R.; Chen, C.-Y.; Hsieh, T.-J.; Cho, C.-P.; Wu, Y.-C. J. Chin. Chemical Soc. (Taipei) 2000, 47, 913. https://doi.org/10.1002/jccs.200000124

6. Yang, Y.-L.; Chang, F.-R.; Wu, Y.-C. Helv. Chim. Acta. 2004, 87, 1392.

https://doi.org/10.1002/hlca.200490127

7. Nkunya, M. H. H.; Jonker, S. A.; Makangara, J. J.; Waibel, R.; Achenbach, H. Phytochemistry 2000, $53,1067$. https://doi.org/10.1016/S0031-9422(00)00012-1

8. Chen, C.-C.; Huang, Y.-L.; Lee, S.-S.; Ou, J.-C. J. Nat. Prod. 1997, 60, 826. https://doi.org/10.1021/np970147c

9. Menachery, M. D.; Blake, G. W.; Gourley, R. C.; Freyer, A. J. Nat. Prod. 1995, 58, 1945. https://doi.org/10.1021/np50126a025

10. Saa, C.; Guitian, E.; Castedo, L.; Suau, R.; Saa, J. M. J Org. Chem. 1986, 51, 2781. https://doi.org/10.1021/jo00364a030

11. Castedo, L.; Saa, C.; Saa, J., M.; Suau, R. J Org. Chem. 1982, 47, 513. https://doi.org/10.1021/jo00342a028

12. Omar, H.; Mohd. Hashim, N.; Zajmi, A.; Nordin, N.; Abdelwahab, S. I.; Azizan, A. H. S.; Hadi, A. H. A.; Mohd Ali, H. Molecules 2013, 18, 8994.

https://doi.org/10.3390/molecules18088994

13. Dhineshkumar, J.; Lamani, M.; Alagiri, K.; Prabhu, K. R. Org. Lett. 2013, 15, 1092. https://doi.org/10.1021/ol4001153

14. Kadam, H. K.; Tilve, S. G. Arkivoc 2015 (vi) 524. http://dx.doi.org/10.3998/ark.5550190.p009.316 
15. Kadam, H. K.; Tilve, S. G. J. Heterocyclic Chem. 2016, 53, 2066. https://doi.org/10.1002/jhet.2213

16. Kadam, H. K.; Malik, D.; Salgaonkar, L.; Mandrekar, K.; Tilve, S. G. Synth. Commun. 2017, 47, 1980. https://doi.org/10.1080/00397911.2017.1359303

17. Nimgirawath, S.; Udomputtimekakul, P. Molecules 2009, 14, 917. https://doi.org/10.3390/molecules14030917

18. Thasana, N.; Bjerke-Kroll, B.; Ruchirawat, S. Synlett 2008 , 4, 505. https://doi.org/10.1055/s-2008-1042764

19. Kuo, R.Y.; Wu, C.C.; Chang, F.R.; Yeh, J.L.; Chen, I.J.; Wu, Y.C. Bioorg. Med. Chem. Lett. 2003, $13,821$. https://doi.org/10.1016/S0960-894X(03)00003-9

20. Szawkalo, J.; Czarnocki, Z., Monat.Chem. 2005, 136, 1619. https://doi.org/10.1007/s00706-005-0341-8

21. Wu, J.; Talwar, D.; Johnston, S.; Yan, M.; Xiao, J. Angew. Chem. Int. Ed. 2013, 52, 6983. https://doi.org/10.1002/anie.201300292

22. Makhey, D.; Gatto, B.; Yu, C.; Liu, A.; Liu, L. F.; LaVoie, E. J. Bioorg. Med. Chem. 1996, 4, 781. https://doi.org/10.1016/0968-0896(96)00054-5

23. Jacobs, J.; Tuyen, N.; Markusse, P.; Stevens, C. V.; Maat, L.; Kimpe, N. Tetrahedron 2009, 65, 1188. https://doi.org/10.1016/i.tet.2008.11.077

24. Castedo, L.; Iglesias, T.; Puga, A.; Saa, J. M.; Suau, R. Heterocycles 1981, 15, 915. https://doi.org/10.3987/S-1981-02-0915

25. Saa, J. M.; Cava, M. P. J Org. Chem. 1978, 43, 1096. https://doi.org/10.1021/jo00400a016 